\title{
Stimulated Brillouin scattering in chalcogenide-PMMA hybrid microwires
}

\author{
J-C. Beugnot ${ }^{1}$, R. Ahmad ${ }^{2}$, M. Rochette ${ }^{2}$, V. Laude ${ }^{1}$, H. Maillotte ${ }^{1}$, T. Sylvestre ${ }^{1}$ \\ ${ }^{1}$ Institut FEMTO-ST, Université de Franche comté, CNRS UMR 6174, Besançon, France \\ ${ }^{2}$ Department of Electrical and Computer Engineering, McGill University, Montreal (QC), Canada
}

\begin{abstract}
We investigate the onset of nonlinear effects in hybrid $\mathrm{As}_{2} \mathrm{Se}_{3}-\mathrm{PMMA}$ microwires and demonstrate that they provide an enhanced Kerr nonlinearity while mitigating stimulated Brillouin scattering when compared to chalcogenide and silica optical fibers.

OCIS codes: $060.4370,190.3270,190.5890$
\end{abstract}

Stimulated Brillouin scattering (SBS) is detrimental to all-optical signal processing using continuous-wave and narrow-linewidth pump lasers. In the last few years, numerous efforts were made to improve the nonlinear (Kerr) figure of merit (FOM) of optical fibers while mitigating the SBS Chalcogenide optical fibers are promising candidates in this regard as they exhibit an ultra-high Kerr nonlinearity and a comparatively lower Brillouin gain increase at $1.55 \mu \mathrm{m}$ [1-6]. In comparison with silica glass, the nonlinear refractive index of $\mathrm{As}_{2} \mathrm{Se}_{3}$ is enhanced $\sim 930$ times while the Brillouin gain coefficient is limited to an enhancement of $\sim 220$ times [4]. In the context of exploiting the Kerr effect without limitation from the Brillouin effect and without axially varying structural characteristics, the overall enhancement in FOM when replacing silica by $\mathrm{As}_{2} \mathrm{Se}_{3}$ can be quantified with the Kerr-toBrillouin FOM is 930/220 = 4,3. In this paper, we investigate the SBS in hybrid $\mathrm{As}_{2} \mathrm{Se}_{3}-\mathrm{PMMA}$ microwires and report a Kerr-to-Brillouin FOM of 110 when replacing the silica fiber by a hybrid microwire, and 27 when replacing an $\mathrm{As}_{2} \mathrm{Se}_{3}$ fiber by a hybrid microwire. We also show that the high nonlinear figure of merit of hybrid microwires relies on the strong Brillouin linewidth broadening due an acoustic wave damping effect of the PMMA coating.

To estimate the SBS limitation, we use the Kerr-to-Brillouin FOM for optical fiber that has recently been introduced by Lee $e t$ al. [8]. This nonlinear figure of merit is written as B-FOM $=\gamma \mathrm{L}_{\text {eff }} \mathrm{P}_{\text {th }}$, where $\gamma$ is the Kerr coefficient, $\mathrm{L}_{\text {eff }}$ is the effective length and $\mathrm{P}_{\mathrm{th}}$ is the SBS power threshold. The $\mathrm{As}_{2} \mathrm{Se}_{3}$ microwires used in the experiments are coated with PMMA, following the procedure detailed in Ref. [1]. The polymer coating adds strength to the otherwise fragile microwire and prevents optical interaction and damage from the outside environment. The microwires are butt-coupled to single-mode fibers (SMF-28) and permanently bonded with UV-epoxy. The microwires have a $13 \mathrm{~cm}$ long uniform section and have a total insertion loss between $4 \mathrm{~dB}$ to $6 \mathrm{~dB}$ for $0.7 \mu \mathrm{m}$ to $1 \mu \mathrm{m}$ core diameter, with $\sim 1 \mathrm{~dB}$ from the Fresnel reflections at the two $\mathrm{As}_{2} \mathrm{Se}_{3}$ to silica interfaces, $\sim 1 \mathrm{~dB}$ propagation loss, and $\sim 1-2 \mathrm{~dB}$ from the mode-mismatch at the input and output ends.

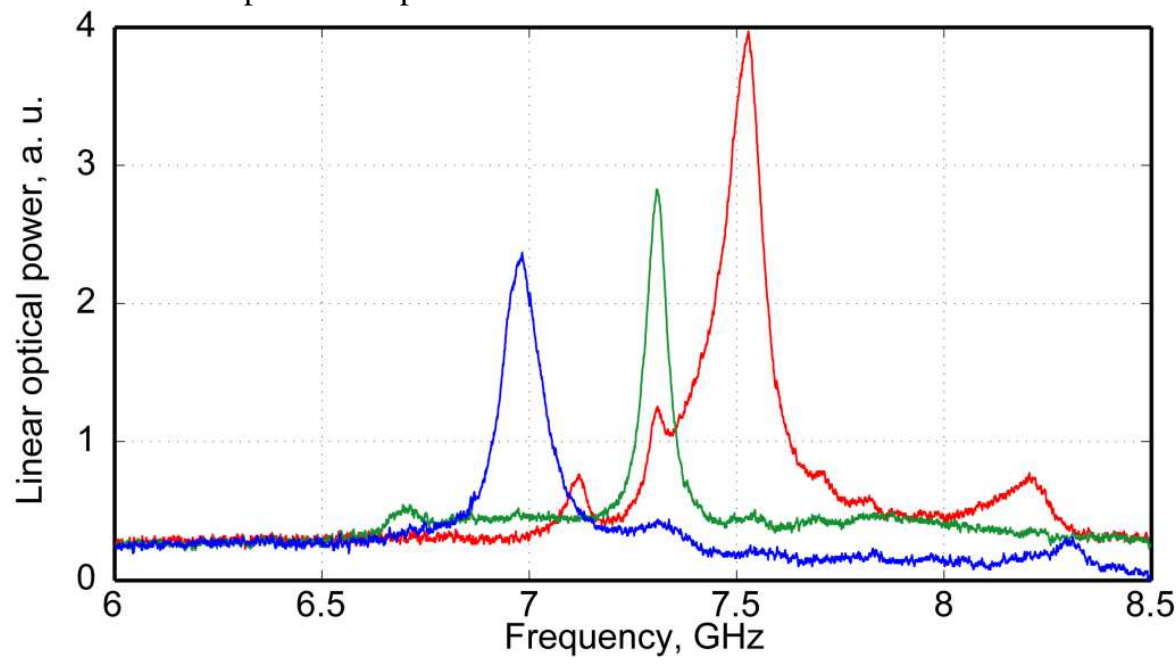

Figure 1: Brillouin spectra measured at a wavelength of $1.55 \mu \mathrm{m}$ in three 13-cm long chalcogenide microwires with a diameter of $1 \mu \mathrm{m}$ (red), 0.9 $\mu \mathrm{m}$ (green) and $0.7 \mu \mathrm{m}$ (blue). The pump power is $1 \mathrm{~mW}$ at the chalcogenide fiber input. On the vertical axis, the total amplitude has been normalized to arbitrary unit but the relative amplitudes between fiber diameters have been conserved. 
SBS measurements were performed with a pump wavelength of $1.55 \mu \mathrm{m}$ and using a standard heterodyne detection [9]. Fig. 1 shows the SBS spectra of three microwires with diameters of $1 \mathrm{um}, 0.9 \mathrm{um}$ and $0.7 \mathrm{um}$. A comparison of the spectra shows that the main SBS frequency peak decreases with the diameter as should be expected from the phase-matching condition and the effective index of the fundamental guided mode. Note also the local peak at a frequency $\sim 8.2 \mathrm{GHz}$ comes from SBS in the $3 \mathrm{~cm}$ long untapered sections of $\mathrm{As}_{2} \mathrm{Se}_{3}$ fiber. For the strongest SBS peaks generated in the wire section of the microwires, the SBS linewidth is $\sim 100 \mathrm{MHz}$, a significantly increased value with respect to the $16 \mathrm{MHz}$ linewidth measured in bulk $\mathrm{As}_{2} \mathrm{Se}_{3}$ [5]. The PMMA cladding surrounding the microwire, with an SBS linewidth of $120 \mathrm{MHz}$, is expected to be responsible of this spectral broadening [2]. This is explained from the larger elastic losses and stronger viscosity of PMMA relative to $\mathrm{As}_{2} \mathrm{Se}_{3}$.

Table 1 compares the linear and nonlinear parameters of standard silica SMF-28, $\mathrm{As}_{2} \mathrm{Se}_{3}$ fibers [5], and the $\mathrm{As}_{2} \mathrm{Se}_{3}$ microwires under test. The $0.7 \mu \mathrm{m}$ microwire exhibits the highest B-FOM, which is 27 times higher than $\mathrm{As}_{2} \mathrm{Se}_{3}$ fibers.

\begin{tabular}{|c|c|c|c|c|c|c|}
\hline & Unit & SMF-28 & $\mathrm{As}_{2} \mathrm{Se}_{3}$ fiber* & Wire1 & Wire2 & Wire3 \\
\hline Core diameter & $\mu \mathrm{m}$ & 10 & 6 & 1 & 0,9 & 0,7 \\
\hline Effective area (@1550nm) & $\mu \mathrm{m}^{2}$ & 85 & 39 & 0,483 & 0,404 & 0,273 \\
\hline Loss (@1550nm) & dB. $m^{-1}$ & 0,0002 & 0,84 & 10 & 10 & 10 \\
\hline Length & m & 1000 & 4,92 & 0,13 & 0,13 & 0,13 \\
\hline Effective length, $L_{\text {eff }}$ & $\mathbf{m}$ & 977,32 & 3,17 & 0,11 & 0,11 & 0,11 \\
\hline Nonlinearity, $\gamma$ & $W^{-1} \cdot m^{-1}$ & 0,001 & 2 & 201 & 241 & 356 \\
\hline Nonlinear coefficient, $\mathrm{n}_{2}$ & $m^{2} \cdot w^{-1}$ & $2,5 \mathrm{E}-20$ & $2,4 \mathrm{E}-17$ & $2,4 \mathrm{E}-17$ & $2,4 \mathrm{E}-17$ & $2,4 \mathrm{E}-17$ \\
\hline Effective index & & 1,44 & 2,81 & 2,59 & 2,54 & 2,37 \\
\hline Brillouin frequency shift & GHz & 11,10 & 7,95 & 7,53 & 7,37 & 6,87 \\
\hline Brillouin linewidth & MHz & 27 & 13,2 & 125 & 65 & 110 \\
\hline Brillouin gain & $\mathrm{m} \cdot \mathrm{W}^{-1}$ & $2,71 \mathrm{E}-11$ & 6,00 E-09 & $3,72 \mathrm{E}-10$ & $6,20 \mathrm{E}-10$ & $2,24 \mathrm{E}-10$ \\
\hline Brillouin threshold, $P_{t h}$ & $\mathrm{dBm}$ & 20,0 & 18,1 & 25,6 & 22,6 & 25,3 \\
\hline K-FOM $\left(\gamma \cdot \mathrm{L}_{\mathrm{eff}}\right)$ & $W^{-1}$ & 1,17 & 7,92 & 22,63 & 27,05 & 40,04 \\
\hline B-FOM $\left(\gamma \cdot L_{\text {eff: }} \cdot P_{\text {th }}\right)$ & & 0,08 & 0,34 & 5,50 & 3,29 & 9,13 \\
\hline
\end{tabular}

Table 1. : Comparison of linear and nonlinear parameters of single-mode fibers, $\mathrm{As}_{2} \mathrm{Se}_{3}$ fibers, and our subwavelength-diameter $\mathrm{As}_{2} \mathrm{Se}_{3}$ microwires with PMMA coating. The measured values are in bold. * ref [5].

To summarize, we have investigated the relative magnitude of nonlinear effects in subwavelength-diameter $\mathrm{As}_{2} \mathrm{Se}_{3}$ microwires and demonstrated a strong increase in the Brillouin threshold compared to $\mathrm{As}_{2} \mathrm{Se}_{3}$ fiber owing to the PMMA coating. This work contributes to the design of a new class of Brillouin-less optical signal processing devices based on the Kerr effect.

This work is supported by the Universite de Franche-Comté, the ANR LABEX ACTION, and the Natural Science and Engineering Research council of Canada (NSERC) .

\section{References}

[1] C. Baker and M. Rochette, "High nonlinearity and single-mode transmission in tapered multi-mode As2Se3-PMMA fibers," IEEE Photonics Journal 4, 960-969 (2012).

[2] Y. Mizuno and K. Nakamura, "Experimental study of Brillouin scattering in perfluorinated polymer optical fiber at telecommunication wavelength," Applied Physics Letters 97, 021103 (2010).

[3] R. Pant et al., "On-chip stimulated Brillouin scattering,” Opt. Exp. 19, 8285-8290 (2011).

[4] K. S. Abedin, "Observation of strong stimulated Brillouin scattering in single-mode $\mathrm{As}_{2} \mathrm{Se}_{3}$ chalcogenide fiber," Opt. Exp. 13, 10266-10271 (2005).

[5] R. Ahmad and M. Rochette, "High Efficiency and ultra broadband optical parametric four-wave mixing in chalcogenide-PMMA hybrid microwires," Opt. Exp. 20, 9572-9580 (2012).

[6] A. Yeniay et al., "Spontaneous and Stimulated Brillouin Scattering Gain Spectra in Optical Fibers," J. Lightwave Technol. 20, 1425-1432 (2002).

[7] R. E. Slusher et al., "Large raman gain and nonlinear phase shifts in high-purity As2Se3 chalcogenide fibers," J. Opt. Soc. Am. B, 21, 1146$1155,(2004)$.

[8] J. H. Lee et al., "Experimental comparison of a Kerr nonlinearity figure of merit including the stimulated Brillouin scattering threshold for state-of-the-art nonlinear optical fibers," Opt. Lett. 30, 1698-1700 (2005).

[9] J-C. Beugnot et al., "Complete experimental characterization of SBS in photonic crystal fiber," Opt. Exp. 15, 15522 (2007). 